\title{
Characterization of non-active site, TrkA selective kinase inhibitors and implications on obtaining kinase selectivity.
}

Hua-Poo Su, Keith Rickert, Christine Burlein, Kartik Narayan, Marina Bukhtiyarova, Danielle M. Hurzy, Craig A. Stump, Xufang Zhang, John C. Reid, Srivanya Tummala, Jennifer M. Shipman, Steven S. Carroll, Stephen M. Soisson, Darrell A. Henze, Andrew J. Cooke

Merck \& Co., Inc.

Current therapies for chronic pain can have insufficient efficacy and lead to side effects, necessitating research on novel targets against pain. Although originally identified as an oncogene, TrkA is linked to pain and elevated levels of NGF, the ligand for TrkA, are associated with chronic pain. Antibodies that block TrkA interaction with its ligand, NGF, are in clinical trials for pain relief. Here, we describe the identification of TrkA-specific inhibitors and the structural basis for their selectivity over other Trk family kinases. The $\mathrm{x}$-ray structures reveal a novel binding site outside the kinase active site that utilizes residues from the kinase domain and the juxtamembrane region. Three modes of binding with the juxtamembrane region are characterized through a series of ligand-bound complexes. The structures indicate a critical pharmacophore on the compounds that leads to the distinct binding modes. The mode of interaction can allow TrkA selectivity over TrkB and TrkC or promiscuous, pan-Trk inhibition. This highlights the difficulty in characterizing the structure-activity relationship of a chemical series in the absence of structural information due to substantial differences in the interacting residues. These structures illustrate the flexibility of binding to sequences outside of, but adjacent to, the kinase domain of TrkA. This knowledge allows development of compounds with specificity for TrkA or the family of Trk proteins and has implications on obtaining selectivity for kinase targets in drug discovery. 